\title{
Évolution de la composition des communautés adventices des cultures de colza sous I'influence des systèmes de culture
}

Guillaume FRIED

Xavier REBOUD

INRA, UMR n' 1210 INRA, Univ. Bourgogne, ENESAD «Biologie et Gestion des Adventices», 17 , rue Sully, BP 86510, 21065 DIJON cedex $<$ reboud@dijon.inra.fr>
Article reçu le 2 août 2006 accepté le 12 janvier 2007

\begin{abstract}
The weed monitoring in France ('Biovigilance Flore') conducted between 2003 and 2005 was used in order to compare the oilseed rape (Brassica napus L.) weed flora with a former weed survey realized in the middle of the 1970's. Using the kind and the degree of specialization of the weed species, this study shows that multiple selective processes operate at various temporal scales, ranging from the crop (one year), to the rotation (about three years) and to decades. Compared to the 1970's, there are more generalist weed species in oilseed rape crops nowadays. In the same time, the number of weed species highly adapted to oilseed rape has increased. About $30 \%$ of the increasing species have been selected by one or several cultivation methods used in oilseed rape culture. But the homogenization of the weed flora could be due to (1) the extension of some rotations including summer crops (maize, sunflower) which could favour the species able to rebuild their soil seed bank every year and (2) the standardization of environmental cropping conditions which could threaten the species highly adapted to particular environments (temporarily waterlogged soils). On a national scale, the soil and the climate are the most effective factors acting on weed species composition. They make the oilseed rape weed flora of the West plains stand out from the flora of the North-East limestone plateau. The effect of interaction between crop rotation and environmental conditions is discussed.
\end{abstract}

Key words: weed flora, Brassica napus L., species turnover, specialist vs. generalist species, new agricultural practices, tillage system

\section{Introduction}

La flore adventice et son évolution sont contraintes à plusieurs échelles temporelles [1]. Par le choix du type de travail du sol, la date de semis, la nature de la culture implantée et les pratiques associées (fertilisation, désherbage), I'agriculteur impose chaque saison culturale un ensemble de perturbations particulières. À moyen terme, la succession de différentes cultures durant la rotation constitue une séquence originale de perturbations qui confère, via les succès et les échecs des espèces, une trajectoire à la communauté de mauvaises herbes hébergées. À ces cycles annuels et pluriannuels s'ajoute à plus long terme l'effet de l'adoption de nouvelles techniques culturales : généralisation du désherbage chimique, nouveaux désherbants sur le marché, modification de la profondeur de travail du sol, etc. La théorie des filtres considère que l'assemblage des espèces en communauté est déterminé par les contraintes environnementales qui agissent comme des filtres sélectionnant les espèces ayant des traits biologiques adaptés [2]. Appliquée à une parcelle cultivée, cette théorie schématise les techniques culturales comme la superposition de plusieurs filtres. Certains sont quasi-identiques tous les ans et définissent les caractéristiques du milieu cultivé tels que perturbations superficielles du sol lors du semis. D'autres peuvent varier chaque année (date de semis, nature des herbicides utilisés) ou évoluer sur le plus long terme (part de l'assolement labouré ou place dédiée à de nouvelles espèces cultivées). Parmi ce dernier type de filtres, les agrosystèmes cultivés ont été marqués durant les 50 dernières années par l'introduction de plusieurs nouvelles espèces cultivées (sorgho, soja) et/ou leur extension sur de plus vastes surfaces (colza, tournesol, maiis). Ceci a eu pour conséquence de modifier profondément les rotations et d'introduire un ensemble de pressions de sélection nouvelles ou différentes sur la flore (date et densité de semis, niveaux de fertilisations, nature des désherbants, date de fermeture du milieu et date de récolte) dont on peut chercher à estimer l'impact.

L'étude de l'évolution de la flore du tournesol a montré qu'au cours de ces 30 dernières années, une très forte sélection s'est exercée sur les communautés adventices, favorisant les espèces ayant des traits biologiques proches du tournesol (grande taille, héliophilie, phénologie calée sur la culture, tolérance aux herbicides utilisées) [3]. Qu'en est-il pour le colza? En 1977, lors du premier inventaire national de la flore adventice [4], le colza avait un historique d'utilisation encore récent sur de nombreuses parcelles échantillonnées. Le retour plus régulier de cette culture dans la rotation amènerait à attendre une spécialisation des communautés adventices du colza. Parallèlement, l'introduction d'autres nouvelles cultures dans les rotations (maïs, tournesol) pourrait favoriser au contraire une homogénéisation de la flore autour de quelques espèces (" passe- 
partout ») ubiquistes. Ainsi, se pose l'alternative suivante : la flore adventice évolue-t-elle plutôt vers la réussite de quelques espèces peu exigeantes qui réussissent quelles que soient les conditions culturales à accroître leur stock de semences (espèces généralistes) ou, au contraire, vers des communautés, composites d'espèces à degré de spécialisation élevée? Les objectifs de cet article se déclinent ainsi en trois étapes : i) mettre en évidence les évolutions majeures de la flore adventice du colza depuis les années 1970, ii) mesurer le degré de spécialisation des espèces par rapport aux conditions environnementales et/ou culturales, iii) estimer le poids des différentes variables sur l'évolution de la composition floristique, à travers leur capacité à différencier les espèces en progression de celles en régression.

\section{Matériels et méthodes}

\section{Données floristiques}

Les données sont issues du réseau Biovigilance Flore, un observatoire permanent de la flore adventice des grandes cultures couvrant toute la France métropolitaine et une gamme étendue de systèmes de cultures [5]. 166 relevés de flore effectués dans des zones témoins non désherbées des parcelles cultivées en colza entre 2003 et 2005 ont pu ainsi être comparés aux 198 relevés effectués dans la même culture entre 1973 et 1976 dans les zones témoins des essais d'homologation des herbicides [4]. Pour chacune des périodes, les relevés de flore ont été réalisés selon la méthode mise au point par $\mathrm{G}$. Barralis [6]. L'ensemble des espèces détectées sur une surface de $2000 \mathrm{~m}^{2}$ est recensé. Un coefficient d'abondance variant de 1 à 5 et exprimant le nombre d'individus observé au $\mathrm{m}^{2}$ (d) est attribué à chaque espèce : $(1) \mathrm{d}<1 / \mathrm{m}^{2} ;(2): 1 \leq \mathrm{d}<3 / \mathrm{m}^{2}$; (3) : $3 \leq \mathrm{d}<20 / \mathrm{m}^{2} ;(4): 20 \leq \mathrm{d}<50 / \mathrm{m}^{2} ;(5): \mathrm{d} \geq 50 / \mathrm{m}^{2}$. Afin de rester homogène avec l'étude des années 1970 , les espèces ayant un coefficient d'abondance inférieur à 2 (qui peuvent être considérées comme accidentelles) ont été éliminées. Ainsi, lorsque la fréquence d'une espèce est égale à $50 \%$, cela signifie que l'espèce a été rencontrée 50 fois avec un cœefficient d'abondance supérieur ou égal à 2 pour 100 relevés effectués [4]. L'échantillonnage de chaque parcelle couvre deux périodes de la campagne culturale : les premières levées d'adventices en automne (octobre/novembre) et la sortie de l'hiver, avant fermeture complète du couvert végétal (février/mars).

Durant les deux périodes d'échantillonnages, l'essentiel des relevés s'est échelonné selon un axe Nord-Est (Moselle-Nord) à Sud-Ouest (CharenteMaritime). Les zones de relevés sont restées représentatives de certains noyaux de production tels que la Marne ou la Vienne, d'autres sont aujourd'hui plus représentés, conformément à l'évolution des surfaces emblavées en colza (Côte d'Or, Deux-Sèvres, Yonne). S'il y a eu une certaine évolution du poids relatif des différentes régions échantillonnées, il n'y a cependant pas eu d'extension de l'aire totale des relevés. Pour tenir compte des différences relatives à la taille de l'échantillon et aux poids variables affectés aux différentes régions, les fréquences des espèces dans les années 1970 sont comparées à l'intervalle de confiance à $5 \%$ calculé autour de la fréquence relative des espèces entre 2003 et 2005. Les données de la première enquête étant uniquement disponibles sous la forme d'une liste synthétique concernant les 26 espèces majeures, les analyses sont restreintes à ces espèces majeures. Le découpage de la France en quatre régions (Nord-Bassin Parisien, Ouest, Sud-Ouest et Est) lors de la première enquête [4] a permis de mesurer l'évolution du statut des espèces aux échelles nationale et régionale.

\section{Données mésologiques}

Pour chaque parcelle échantillonnée entre 2003 et 2005, des questionnaires remplis par les agriculteurs donnent accès aux conditions environnementales de la parcelle et aux pratiques culturales qui y ont été conduites. En raison de données manquantes, seules 98 parcelles et 12 variables ont servit à établir les corrélations entre flore adventice, milieu physique et techniques culturales pour la période 2003-2005 (tableau 1). Les variables concernant les herbicides montrant une gamme étendue de modalités, n'ont été utilisées dans les analyses multifactorielles qu'en variables supplémentaires. Le mode de travail du sol oppose la pratique du non-labour (semis-direct) et les techniques culturales simplifiées (TCS) (semis uniquement précédé d'un passage de herse) au labour conventionnel avec retournement d'une couche de sol. La connaissance de la profondeur maximale de travail du sol constitue une mesure de l'intensité de ces perturbations. À partir des coordonnées géographiques de la parcelle (longitude, latitude, altitude), les données climatiques (température minimale et précipitation) de Météo-France ont été obtenues par la méthode d'interpolation Aurelhy [7] sur la moyenne des trois dernières décennies complètes (1971-2000).

\section{Analyse de la nature et du degré de spécialisation des espèces}

Afin de mesurer l'effet des facteurs agro-écologiques sur la flore adventice, trois indices de spécialisation ont été calculés. Le premier indice reflète l'amplitude écologique des espèces (Sgene), tous facteurs confondus (i.e. par rapport au type de culture, au type de sol, à la région climatique, etc.), un second indice donne plus précisément le degré d'affinité des espèces aux différents types de culture (Scrop) et enfin, un troisième indice exprime la spécialisation des espèces au sein de la diversité des situations agro-écologiques existant dans les cultures de colza (Srape).

1) Sgene : l'amplitude écologique des espèces a été obtenu en calculant le pouvoir indicateur des espèces en suivant la méthodologie mise au point par Brisse et al. [8]. Cet indice indépendant de toutes variables du milieu, est basé sur la notion de fidélité des espèces entre elles. II permet sur cette base de distinguer simplement les espèces spécialistes qui se trouvent statistiquement associées à un ensemble restreint d'espèces (Sgene élevé) de celles, plus généralistes, qui ont une distribution plus aléatoire, avec pour conséquence un lien statistique faible avec un ensemble étendu d'espèces (Sgene faible).

2) Scrop : Pour estimer le degré d'affinité par rapport au type de culture, nous avons utilisé la procédure IndVal [9]. Cette méthode permet de calculer la valeur indicatrice (IV) des espèces par rapport à des groupes de relevés, issus dans le cas présent d'une classification non hiérarchique définie volontairement par le type de culture. La valeur indicatrice des 243 espèces adventices présentes dans 1554 relevés (issus du réseau Biovigilance Flore) a donc été calculée en fonction de leurs fréquences et de leurs abondances au sein des cultures rangées en sept catégories: 1-céréales d'hiver ; 2-colza ; 3-céréales de printemps ; 4-protéagineux ; 5-betteraves; 6-tournesol/soja et 7-maïs/sorgho.

L'indice IV combine pour chaque espèce $i$, une valeur d'abondance relative $(A)$ et de fréquence relative $(B)$ par rapport à chaque groupe de stations occupées par la culture $j:$ IV $=\left(A_{i j} / A_{i}\right) \times\left(B_{i j} / B_{. j}\right) \times 100$. Ces deux valeurs représentant des informations indépendantes, sont multipliées. L'indice est ensuite exprimé en pourcentage. Le cas extrême d'un indice de $100 \%$ pour une culture signifie que l'on trouve une espèce dans tous les relevés réalisés dans cette culture et qu'elle est en même temps absente de tous les relevés des autres cultures. La significativité des valeurs indicatrices est mesurée au moyen d'un test de permutation de Monte Carlo (source) réalisé par 1000 permutations aléatoires.

3) Afin de préciser la nature de la spécialisation des espèces au sein des grands types de situations pédo-climatiques et d'itinéraires culturaux dans le colza (Srape), une analyse multivariée permettant de mesurer la corrélation entre matrice floristique et mésologique a été réalisée. En raison de la nature à la fois qualitative et quantitative des variables du 
Tableau 1. Caractéristiques des 4 principales situations agro-écologiques des parcelles de colza. Moyennes et intervalles de confiance (à $5 \%$ ) des variables quantitatives et pourcentage de chaque modalité des variables qualitatives.

\begin{tabular}{|c|c|c|c|c|c|c|c|c|}
\hline Variables & \multicolumn{2}{|c|}{$\mathbf{I}$} & \multicolumn{2}{|c|}{ II } & \multicolumn{2}{|c|}{ III } & \multicolumn{2}{|c|}{ IV } \\
\hline Variables qualitatives & \multicolumn{2}{|c|}{$\%$} & \multicolumn{2}{|c|}{$\%$} & \multicolumn{2}{|c|}{$\%$} & \multicolumn{2}{|c|}{$\%$} \\
\hline \multicolumn{9}{|l|}{ Texture du sol } \\
\hline Argileux & \multicolumn{2}{|c|}{63} & \multicolumn{2}{|c|}{22} & \multicolumn{2}{|c|}{23} & \multicolumn{2}{|c|}{50} \\
\hline Argilo-limoneux & \multicolumn{2}{|c|}{11} & \multicolumn{2}{|c|}{14} & \multicolumn{2}{|c|}{14} & \multicolumn{2}{|c|}{00} \\
\hline Limoneux & \multicolumn{2}{|c|}{11} & \multicolumn{2}{|c|}{14} & \multicolumn{2}{|c|}{45} & \multicolumn{2}{|c|}{14} \\
\hline Limono-argileux & \multicolumn{2}{|c|}{04} & \multicolumn{2}{|c|}{32} & \multicolumn{2}{|c|}{18} & \multicolumn{2}{|c|}{07} \\
\hline Sablonneux & \multicolumn{2}{|c|}{11} & & 8 & & & & \\
\hline Type de travail du sol & & & & & & & & \\
\hline TCS & & & & 1 & & & & \\
\hline Labour conventionnel & & & & 9 & & & & \\
\hline Précédent cultural & & & & & & & & \\
\hline Blé hiver & & & & 0 & & & & \\
\hline Orge d’hiver & & & & 15 & & & & \\
\hline Orge de printemps & & & & 5 & & & & \\
\hline Antécédent cultural & & & & & & & & \\
\hline Céréales d'hiver & & & & 36 & & & & \\
\hline Cultures estivales (maïs, tournesol) & & & & 4 & & & & \\
\hline Variables quantitatives & Moy. & Int. $5 \%$ & Moy. & Int. $5 \%$ & Moy. & Int. $5 \%$ & Moy. & Int. $5 \%$ \\
\hline Latitude & 48,38 & 0,33 & 47,50 & 0,30 & 47,95 & 0,40 & 46,47 & 0,51 \\
\hline Longitude & 3,95 & 0,28 & 4,38 & 0,36 & 1,74 & 0,42 & 0,26 & 0,49 \\
\hline Altitude (en m) & 164,89 & 17,47 & 225,78 & 16,71 & 122,05 & 12,56 & 130,82 & 29,37 \\
\hline Température minimale $\left(\mathrm{en}{ }^{\circ} \mathrm{C}\right.$ ) & $-0,75$ & 0,19 & $-1,22$ & 0,26 & 0,45 & 0,15 & 1,11 & 0,26 \\
\hline Précipitation annuelle (mm) & 733,61 & 15,71 & 796,46 & 28,32 & 707,39 & 14,92 & 749,97 & 44,48 \\
\hline $\mathrm{pH}$ du sol & 7,26 & 0,21 & 6,89 & 0,12 & 7,08 & 0,17 & 6,97 & 0,20 \\
\hline Date de semis $\left(0=1^{\mathrm{er}}\right.$ janvier $\left.)\right)$ & $-130,13$ & 2,10 & $-127,90$ & 1,96 & $-125,43$ & 1,95 & $-120,26$ & 2,80 \\
\hline Profondeur de labour (en m) & 0,16 & 0,03 & 0,04 & 0,03 & 0,20 & 0,02 & $\mathbf{0 , 1 0}$ & 0,04 \\
\hline Surface de la parcelle (en ha) & 7,57 & 2,34 & 9,67 & 2,99 & 12,92 & 3,24 & 8,01 & 1,71 \\
\hline Désherbage & Moy. & Int. $5 \%$ & Moy. & Int. $5 \%$ & Moy. & Int. $5 \%$ & Moy. & Int. $5 \%$ \\
\hline Nomb. de familles chimiques utilisées & 2,24 & 0,38 & 2,74 & 0,32 & 2,08 & 0,37 & 2,28 & 0,46 \\
\hline Nomb. de mat. act. par passage & 1,99 & 0,37 & 2,20 & 0,34 & 1,88 & 0,36 & 1,87 & 0,42 \\
\hline Nomb. de passages & 1,72 & 0,38 & 1,87 & 0,33 & 1,62 & 0,26 & 1,67 & 0,33 \\
\hline$\%$ de la dose homologuée & 0,82 & 0,07 & 0,85 & 0,05 & 0,90 & 0,08 & 0,82 & 0,07 \\
\hline Indices de diversité & Moy. & Int. $5 \%$ & Moy. & Int. $5 \%$ & Moy. & Int. $5 \%$ & Moy. & Int. $5 \%$ \\
\hline Densité moyenne (plantes/m²) & 23,17 & 6,46 & 34,57 & 6,45 & 30,93 & 7,24 & 45,10 & 8,45 \\
\hline Richesse spécifique & 8,17 & 2,68 & 11,83 & 2,08 & 11,36 & 2,55 & 15,10 & 2,81 \\
\hline Diversité (Indice de Shannon) & 2,52 & 0,43 & 3,34 & 0,25 & 3,05 & 0,42 & 3,67 & 0,25 \\
\hline Equitabilité & 0,967 & 0,010 & 0,974 & 0,005 & 0,975 & 0,008 & 0,967 & 0,009 \\
\hline
\end{tabular}

milieu (tableau 1), nous avons dans un premier temps procédé à une analyse dite de «Hill \& Smith» [10]. L'analyse de «Hill \& Smith» autorise le mélange d'une analyse en composantes principales (ACP) normée sur variables quantitatives et d'une analyse des correspondances multiples (ACM) sur variables qualitatives. Une classification ascendante hiérarchique $(\mathrm{CAH})$ a ensuite été réalisée sur les coordonnées de l'analyse «Hill \& Smith » avec la méthode d'agrégation de Ward [11]. Ce traitement regroupe donc de manière hiérarchique les parcelles les plus homogènes du point de vue des variables agro-écologiques considérées. Dans un second temps, la même procédure IndVal a été utilisée pour calculer la valeur indicatrice (IV) des 128 espèces présentes pour chaque partition de la $\mathrm{CAH}$; une espèce répartie dans toutes les conditions étant bien sûr moins spécialisée qu'une espèce concentrée sur un nombre restreint de partitions. Enfin, pour préciser les relations entre les espèces et les variables agro-écologiques, une analyse de co-inertie [12] a été réalisée entre les triplets statistiques mésologiques (issue de l'analyse Hill
\& Smith) et floristiques (issue d'une ACP). Toutes les analyses statistiques multivariées ont été réalisées à l'aide du logiciel Ade-4 [13].

\section{Résultats}

\section{Évolution de la flore}

Les 26 espèces majeures des deux périodes se répartissent en 16 familles taxonomiques. Les quatre familles botaniques les mieux représentées constituent plus de la moitié des espèces majeures $(53,13 \%)$ : Poacées (6 espèces), Astéracées (5 espèces), Brassicacées (4 espèces) et Caryophyllacées ( 3 espèces). Les communautés sont très largement dominées par des espèces annuelles: le pourcentage d'espèces vivaces est faible (4\%) et reste stable entre les deux périodes, avec la régression d'Elytrigia repens compensée par la progression de Cirsium arvense. La proportion de Poacées, les 26 espèces les plus fréquentes, a diminué passant de 
$27 \%$ à $12 \%$. Parmi les thérophytes, la proportion d'espèces estivales est passée d'une à quatre espèces. Le tableau 2 donne les rangs et les fréquences relatives des 50 principales espèces adventices pour les deux périodes de relevés ainsi que leur tendance évolutive aux échelles nationale et régionale.

1) À l'échelle de la France, sept espèces ont fait leur entrée au sein des 26 espèces classées comme majeures sur la base des fréquences. Cela correspond à un changement majeur de statut en 30 ans pour des espèces comme Geranium dissectum, Sonchus asper et Chenopodium album. Très rares dans les cultures de colza durant les années 1970 (fréquences quasi nulles, inférieures à 3,6 \%), ces espèces se développent aujourd'hui dans 15 à $17 \%$ des parcelles. Dans une moindre mesure, c'est également le cas de Lapsana communis, Solanum nigrum, Cirsium arvense et Polygonum persicaria, qui ont au moins doublé leur fréquence dans les cultures de colza avec une présence actuelle dans environ $9 \%$ des parcelles. Sont également dans ce cas de figure, des espèces comme Senecio vulgaris, Lolium spp. ou Mercurialis annua qui bien que figurant déjà parmi les 26 espèces majeures dans les années 1970, passent de fréquences faibles comprises entre 4 et $7 \%$ des parcelles à des fréquences importantes autour de $14-26 \%$ voire $20-33 \%$ pour $M$. annua. Des espèces déjà très abondantes il y a 30 ans ont continué à progresser et dominent aujourd'hui la flore du colza : Sinapis arvensis, Viola arvensis. À l'opposé, Alopecurus myosuroides et Papaver rhoeas ont régressé mais restent à des fréquences élevées, tandis que Avena fatua est en déclin plus marqué. Des espèces peu fréquentes dans les années 1970 se raréfient encore un peu plus : Elytrigia repens, Arabidopsis thaliana et Avena sterilis. ssp. ludoviciana. Enfin, Aphanes arvensis, Matricaria recutita, Matricaria perforata, Stellaria media, Veronica hederifolia et Veronica persica sont en régression tout en maintenant un niveau de fréquence intermédiaire proche de $10 \%$

2) À l'échelle régionale, la progression de certaines espèces se limite soit aux régions des plateaux calcaires de l'Est (Geranium rotundifolium, Cirsium arvense, Lapsana communis, Aethusa cynapium) soit aux plaines de l'Ouest (Euphorbia helioscopia, Polygonum persicaria, Polygonum aviculare, Anagallis arvensis). Alopecurus myosuroides demeure la principale espèce adventice des colzas de l'Est, tandis que dans I'Ouest, $M$. annua est devenue l'adventice majeure des cultures de colza au cours des 30 dernières années.

\section{Nature et degré de spécialisation des espèces}

\section{Amplitude écologique (Sgene)}

La comparaison des listes des 26 adventices les plus fréquentes entre les périodes 1973-76 et 2003-05 montre que, de nos jours, le colza héberge des espèces plus ubiquistes qu'il y a 30 ans, avec un degré de spécialisation moyen passant de $\operatorname{Sgen}_{1970}=4,17(+/-0,36)$ à Sgen $_{2000}=3,84$ $(+/-0,19)$. Cette évolution est en limite de significativité (test de Student, $p=0,06$ ) mais est cependant beaucoup plus marquée dans les plaines de I'Ouest où le nombre d'espèces généralistes en progression est plus important, faisant significativement baisser le degré de spécialisation moyen des 26 espèces majeures de $\mathrm{Sg}_{1970}=4,20$ à $\mathrm{Sg}_{2000}=3,76$ $(p<0,05)$. Les espèces en progression sont significativement plus généralistes que celles en régression ( $p<0,05$, quelles que soient l'échelle et la région considérée). Les espèces stables ont aussi un degré de spécialisation intermédiaire.

\section{Degré d'affinité à la culture (Scrop)}

Sur les 243 espèces détectées dans le réseau «Biovigilance Flore », 20 sont indicatrices de la culture du colza (soit un cinquième des espèces détectées dans cette culture), dont 5 Brassicacées et 3 Géraniacées (tableau 3). Seules $40 \%$ de ces espèces spécifiques du colza sont des espèces majeures de cette culture au niveau national (rang $<25$, $\mathrm{F}>8 \%) ; 45 \%$ restent des adventices localisées, peu fréquentes au niveau national (rang $>50 ; \mathrm{F}<2,5 \%$ : Thlaspi arvense, Scandix pectenveneris, Anchusa arvensis) et $15 \%$ présentent un statut intermédiaire (Geranium rotundifolium, Bromus sterilis et Calepina irregularis). Le nombre d'espèces inféodées au colza a doublé au sein des 26 espèces les plus fréquentes de cette culture, passant de 16 à $32 \%$ des espèces. Ces espèces représentent ainsi $31 \%$ des espèces en progression. Parallèlement, on assiste à une forte régression des spécialistes des céréales $(64 \%$ des espèces en régression). Dans le même temps, le nombre de spécialistes des «nouvelles cultures » de printemps (maïs, tournesol) a été multiplié par quatre (passant de 1 à 4 espèces parmi les 26 plus fréquentes dans le colza).

\section{Degré de spécialisation aux situations agro-écologiques des cultures de colza (Srape)}

La CAH effectuée sur les 12 variables agro-écologiques considérées permet de distinguer quatre grands types de situations (notés I, II, III et IV) dont les caractéristiques mésologiques sont résumées dans le tableau 1. Pour un cinquième des parcelles analysées, nous disposons de trois années de recul sur la rotation (tableau 1). L'analyse des successions culturales montre alors clairement qu'au-delà du précédent cultural (qui est toujours une céréale), les groupements II et IV se distinguent très nettement par le type de rotation. $86 \%$ des parcelles du groupe II sont des parcelles où le colza s'insère dans une rotation de type « colza-bléorge » alors que cette proportion se réduit à $29 \%$ dans le groupe IV où $71 \%$ des colzas appartiennent à une rotation de type «céréales(tournesol ou maïs)-céréales-colza ».

Les variations de la fréquence et de l'abondance de certaines espèces ne sont pas significativement différentes entre les quatre groupes de parcelles (tableau 4): Viola arvensis, Sinapis arvensis, Lolium spp., Raphanus raphanistrum, Myosotis arvensis et Lapsana communis. D'autres espèces apparaissent en revanche comme bien plus inféodées à certaines situations: Geranium dissectum, Alopecurus myosuroides, Geranium rotundifolium, Bromus sterilis, Cirsium arvense et Geranium molle sont significativement indicatrices du groupe II (région Centre-Est, plateau, travail du sol superficiel, rotation "colza-blé-orge") tandis que Mercurialis annua, Senecio vulgaris et Chenopodium album sont liées au groupe IV (région Centre-Ouest, plaine, climat doux en hiver, rotations incluant une ou plusieurs cultures estivales). Les conditions environnementales des groupes I et III ne semblent pas développer une flore indicatrice marquée. L'analyse de co-inertie (résultats non présentés) confirme ces résultats et permet de préciser certaines relations espèces-milieu. Ainsi, les parcelles conduites en non-labour sont caractérisées par des abondances significativement plus élevées de repousses d'orge (Hordeum vulgare) et des adventices Sinapis arvensis, Viola arvensis, Bromus sterilis, Geranium dissectum et Capsella bursa-pastoris, tandis que les parcelles où les labours sont les plus profonds sont dominées par Chenopodium album, Apera spicaventi, Lamium purpureum et Polygonum persicaria.

\section{Discussion}

La nature hétérogène et incomplète des données tout comme la focalisation sur les 26 espèces les plus communes peut avoir conduit à appréhender diversement le poids de certains facteurs dans l'évolution de la flore exprimée dans la culture de colza. Nos résultats montrent cependant que les techniques d'ordination et de classification de la végétation semblent suffisamment robustes pour extraire les principales tendances agro-écologiques sous-jacentes aux changements de statut des espèces. En intégrant en particulier la dimension spécialiste/ ubiquiste de la flore rencontrée pour différents facteurs, cette analyse illustre l'intérêt d'étudier l'évolution des communautés adventices à différentes échelles emboîtées pour saisir la complexité des processus en jeu. 
Tableau 2. Évolution de la fréquence entre 1973-1976 et 2002-2005, amplitude écologique (Sgene) et degré d'affinité avec les cultures (Scrop) des principales espèces adventices du colza.

\begin{tabular}{|c|c|c|c|c|c|c|c|}
\hline \multirow[t]{2}{*}{ Espèces } & \multicolumn{2}{|r|}{2005} & \multicolumn{2}{|c|}{1975} & \multirow{2}{*}{$\begin{array}{l}\text { Evolution }^{\mathrm{b}} \\
\text { Nationale } \\
\text { (Ouest/Est) }\end{array}$} & \multirow{2}{*}{$\begin{array}{c}\text { Sgene } \\
\text { Degré de } \\
\text { spécialisation }\end{array}$} & \multirow{2}{*}{$\begin{array}{c}S_{\text {crop }}{ }^{c} \\
\text { (Lien à une culture) }\end{array}$} \\
\hline & Rang & $\begin{array}{c}\text { Fréq. } \%{ }^{a} \\
\text { (min-max) }\end{array}$ & Rang & Fréq. & & & \\
\hline Triticum spp. & 1 & $30,1(23-37,1)$ & $?$ & $?$ & $?(? / ?)$ & 3,97 & Colza $\left(25,23^{* *}\right)$ \\
\hline Viola arvensis & 2 & $28,2(21,3-35,1)$ & 10 & 19,7 & $+(+/+)$ & 3,68 & CerHiv $\left(9,11^{* *}\right)$ \\
\hline Capsella bursa-pastoris & 3 & $27,6(20,7-34,5)$ & 8 & 21,7 & $=(+/+)$ & 3,43 & Colza $\left(13,75^{\star *}\right)$ \\
\hline Sinapis arvensis & 3 & $27,6(20,7-34,5)$ & 11 & 19,2 & $+(+/+)$ & 3,76 & CerPri $\left(11,02^{* *}\right)$ \\
\hline Alopecurus myosuroides & 5 & $27(20,2-33,8)$ & 1 & 52,1 & $-(-/=)$ & 4,11 & CerPri $\left(8,79^{* *}\right)$ \\
\hline Mercurialis annua & 6 & $26,4(19,6-33,1)$ & 23 & 4,6 & $+(+/+)$ & 3,56 & Bett. $\left(15,13^{* *}\right)$ \\
\hline Senecio vulgaris & 7 & $20,9(14,6-27,1)$ & 19 & 6,6 & $+(+/+)$ & 3,19 & Tournesol $\left(11,00^{* *}\right)$ \\
\hline Lolium spp. & 8 & $19,6(13,5-25,7)$ & 17 & 6,6 & $+(+/+)$ & 3,41 & Colza $\left(8,34^{* *}\right)$ \\
\hline Galium aparine & 9 & $19(13-25)$ & 9 & 20,7 & $=(=/=)$ & 3,88 & CerPri $\left(10,43^{* *}\right)$ \\
\hline Hordeum spp. & 10 & $19(13-25)$ & $?$ & $?$ & $?(? / ?)$ & 4,57 & Colza $\left(25,77^{* *}\right)$ \\
\hline Papaver rhoeas & 11 & $17,8(11,9-23,7)$ & 5 & 26,8 & $-(=/=)$ & 4,09 & CerPri $\left(8,79^{* *}\right)$ \\
\hline Geranium dissectum & 12 & $17,2(11,4-23)$ & $>26$ & $<3,6$ & $N(N / N)$ & 4,10 & Colza $\left(28,04^{* *}\right)$ \\
\hline Sonchus asper & 13 & $16(10,3-21,6)$ & $>26$ & $<3,6$ & $N(N / N)$ & 3,25 & Tournesol $\left(7,35^{\star \star}\right)$ \\
\hline Chenopodium album & 14 & $15,3(9,8-20,9)$ & $>26$ & $<3,6$ & $\mathrm{~N}(\mathrm{~N} / \mathrm{N})$ & 3,21 & Maïs $\left(24,38^{* *}\right)$ \\
\hline Matricaria recutita + perforata & 15 & $14,1(8,8-19,5)$ & 4 & 31,3 & $-(=/=)$ & 3,85 et 3,93 & Prot. $\left(6,22^{*}\right)$ et Bett. $\left(2,99^{*}\right)$ \\
\hline Raphanus raphanistrum & 15 & $14,1(8,8-19,5)$ & 13 & 15,2 & $=(=/+)$ & 3,84 & Colza $\left(5,78^{* *}\right)$ \\
\hline Myosotis arvensis & 17 & $13,5(8,3-18,7)$ & 18 & 6,6 & $+(+/+)$ & 4,54 & Colza $\left(8,54^{* *}\right)$ \\
\hline Poa annua & 18 & $12,3(7,2-17,3)$ & 12 & 16,7 & $=(+/-)$ & 3,59 & CerHiv $\left(11,15^{\star *}\right)$ \\
\hline Aphanes arvensis & 19 & $11,7(6,7-16,6)$ & 7 & 23,7 & $-(=/-)$ & 5,21 & CerHiv $\left(8,95^{\star *}\right)$ \\
\hline Veronica hederifolia & 20 & $11(6,2-15,9)$ & 6 & 26,3 & $-(-/=)$ & 4,45 & CerHiv $(14,89 * *)$ \\
\hline Solanum nigrum & 21 & $9,8(5,2-14,4)$ & $>26$ & $<3,6$ & $\mathrm{~N}(? / \mathrm{N})$ & 3,49 & Maïs $(22,19 * *)$ \\
\hline Stellaria media & 21 & $9,8(5,2-14,4)$ & 2 & 49,5 & $-(-/-)$ & 3,52 & CerHiv $\left(10,71^{* *}\right)$ \\
\hline Lapsana communis & 23 & $9,2(4,8-13,6)$ & $>26$ & $<3,6$ & $\mathrm{~N}(? / \mathrm{N})$ & 4,07 & Colza $\left(4,55^{\star *}\right)$ \\
\hline Cirsium arvense & 24 & $8,6(4,3-12,9)$ & $>26$ & $<3,6$ & $\mathrm{~N}(? / \mathrm{N})$ & 3,44 & Bett. $\left(7,64^{* *}\right)$ \\
\hline Lamium purpureum & 24 & $8,6(4,3-12,9)$ & 21 & 5,1 & $=(+1=)$ & 3,80 & - \\
\hline Polygonum persicaria & 24 & $8,6(4,3-12,9)$ & $>26$ & $<3,6$ & $\mathrm{~N}(\mathrm{~N} / ?)$ & 3,51 & Maïs $\left(11,87^{\star *}\right)$ \\
\hline Veronica persica + polita & 27 & $9,2(4,8-13,6)$ & 3 & 31,8 & $-(=/-)$ & 3,65 et 4,52 & CerHiv $(7,89 * *)$ \\
\hline Fumaria officinalis & 28 & $8(3,8-12,1)$ & 16 & 9,1 & $=(+/-)$ & 3,82 & CerPri $\left(8,38^{* *}\right)$ \\
\hline Euphorbia helioscopia & 29 & $7,4(3,4-11,4)$ & $>26$ & $<3,6$ & $?(\mathrm{~N} / ?)$ & 3,67 & Tournesol $\left(4,70^{*}\right)$ \\
\hline Sonchus oleraceus & 29 & $7,4(3,4-11,4)$ & $>26$ & $<3,6$ & $?(? / ?)$ & 3,55 & - \\
\hline Ranunculus sardous & 31 & $6,7(2,9-10,6)$ & $>26$ & $<3,6$ & $?(? / ?)$ & 4,47 & - \\
\hline Anagallis arvensis & 32 & $6,1(2,5-9,8)$ & $>26$ & $<3,6$ & $?(\mathrm{~N} / ?)$ & 3,37 & - \\
\hline Geranium rotundifolium & 32 & $6,1(2,5-9,8)$ & $>26$ & $<3,6$ & $?(? / \mathrm{N})$ & 3,94 & Colza $\left(3,47^{\star *}\right)$ \\
\hline Polygonum aviculare & 32 & $6,1(2,5-9,8)$ & $>26$ & $<3,6$ & $?(\mathrm{~N} / ?)$ & 3,19 & CerPri $\left(12,43^{* *}\right)$ \\
\hline Convolvulus arvensis & 35 & $5,5(2-9)$ & $>26$ & $<3,6$ & $?(? / ?)$ & 3,57 & Tournesol $\left(11,93^{* *}\right)$ \\
\hline Daucus carota & 35 & $5,5(2-9)$ & $>26$ & $<3,6$ & $?(? / ?)$ & 3,71 & Tournesol $\left(5,57^{* *}\right)$ \\
\hline Bromus sterilis & 37 & $4,9(1,6-8,2)$ & $>26$ & $<3,6$ & $?(? / ?)$ & 4,34 & Colza $\left(4,67^{* *}\right)$ \\
\hline Picris hieracioides & 38 & $4,3(1,2-7,4)$ & $>26$ & $<3,6$ & $?(? / ?)$ & 4,38 & - \\
\hline Aethusa cynapium & 39 & $3,7(0,8-6,6)$ & $>26$ & $<3,6$ & $?(? /+)$ & 4,31 & Bett. $\left(10,32^{\star \star}\right)$ \\
\hline Chenopodium hybridum & 39 & $3,7(0,8-6,6)$ & $>26$ & $<3,6$ & $?(? / ?)$ & 4,46 & - \\
\hline Chenopodium polyspermum & 39 & $3,7(0,8-6,6)$ & $>26$ & $<3,6$ & $?(? / ?)$ & 4,47 & Maïs $\left(6,64^{* *}\right)$ \\
\hline Calepina irregularis & 39 & $3,7(0,8-6,6)$ & $>26$ & $<3,6$ & $?(? / ?)$ & 5,84 & Colza $\left(3,62^{* *}\right)$ \\
\hline Poa trivialis & 39 & $3,7(0,8-6,6)$ & 22 & 4,72 & $?(=/=)$ & 4,12 & - \\
\hline Taraxacum officinale & 39 & $3,7(0,8-6,6)$ & $>26$ & $<3,6$ & $?(? / ?)$ & 3,45 & - \\
\hline Veronica arvensis & 39 & $3,7(0,8-6,6)$ & $>26$ & $<3,6$ & $?(? / ?)$ & 4,13 & - \\
\hline Cerastium spp. & 39 & $3,7(0,8-6,6)$ & 20 & 5,6 & $=(=/=)$ & 7,97 et 4,50 & CerHiv $\left(4,63^{* *}\right)$ \\
\hline Lactuca serriola & 47 & $3,1(0,4-5,7)$ & $>26$ & $<3,6$ & $?(? / ?)$ & 4,00 & - \\
\hline Ammi majus & 48 & $2,5(0,1-4,8)$ & $>26$ & $<3,6$ & $?(? / ?)$ & 4,49 & Tournesol $\left(3,45^{\star *}\right)$ \\
\hline Arenaria serpyllifolia & 49 & $2,5(0,1-4,8)$ & $>26$ & $<3,6$ & $?(? / ?)$ & 5,97 & - \\
\hline Avena fatua & 50 & $2,5(0,1-4,8)$ & 14 & 12,1 & $-(=/-)$ & 4,10 & Prot. $(10,79 * *)$ \\
\hline Elytrigia repens & 65 & $1,2(0-2,9)$ & 25 & 3,6 & $-(? / ?)$ & 3,77 & - \\
\hline Arabidopsis thaliana & 80 & $0,6(0-1,8)$ & 24 & 4,5 & $-(-/=)$ & 4,90 & CerHiv $\left(2,61^{*}\right)$ \\
\hline Avena sterilis subsp. Iudoviciana & $>100$ & $0(0-0)$ & 15 & 9,6 & $-(-/ ?)$ & 7,26 & - \\
\hline Spergula arvensis & $>100$ & $0(0-0)$ & 26 & 3,6 & $-(-/-)$ & 4,51 & Maïs $(4,30 * *)$ \\
\hline
\end{tabular}

* significatif à $\mathrm{p}<0.05 ;{ }^{* *}$ significatif à $\mathrm{p}<0.01$.

a Les espèces accidentelles ayant un coefficient d'abondance inférieur à 2 n'étant pas considérées dans le calcul de la fréquence, une fréquence de 50 \% signifie que l'espèce a été rencontrée 50 fois avec un cœfficient d'abondance supérieur ou égal à 2 pour 100 relevés effectués.

b ' + ' : en progression ; ' $=$ ' stable ; '-' : en régression ; $N$ ' : nouveau parmi les 26 espèces majeures.

${ }^{c}$ CerHiv = céréales d'hiver, CerPri = céréale de printemps, Prot = protéagineux, Bett = betterave. 
Tableau 3. Liste des espèces significativement indicatrices de la culture de colza. Les notes de sensibilité aux herbicides du colza ont été recalculées à partir des données compilées par Mamarot et Rodriguez [17]. Les codes couleurs reflétant la sensibilité des espèces pour chaque formulation commerciale ont été traduits en variables ordinales de 1 (efficacité inférieure à $70 \%$ ) à 7 (efficacité au moins supérieure à $95 \%$ ). Pour chaque espèce, la valeur moyenne par rapport aux herbicides utilisés en colza a été ramenée sur une base 100. Ainsi, une espèce qui aurait un indice de sensibilité de $100 \%$ serait très sensible à tous les herbicides du colza tandis qu'une espèce ayant un indice de $0 \%$ ne serait contrôlée par aucun de ces herbicides.

\begin{tabular}{|c|c|c|c|}
\hline Espèces & Famille & $\begin{array}{c}S_{\text {crop }} \\
\text { (IndVal) }\end{array}$ & $\begin{array}{l}\text { Sensibilité } \\
\text { aux herbicides } \\
\text { du colza }\end{array}$ \\
\hline Geranium dissectum & Geraniaceae & $28,04^{\star *}$ & 30,00 \\
\hline Capsella bursa-pastoris & Brassicaceae & $13,75^{\star *}$ & 36,36 \\
\hline Myosotis arvensis & Boraginaceae & $8,54^{* *}$ & 66,67 \\
\hline Lolium spp. & Poaceae & $8,34^{* *}$ & 81,11 \\
\hline Raphanus raphanistrum & Brassicaceae & $5,78^{* *}$ & 30,30 \\
\hline Bromus sterilis & Poaceae & $4,67^{* *}$ & 68,18 \\
\hline Lapsana communis & Asteraceae & $4,55^{* *}$ & 50,00 \\
\hline Valerianella spp. & Valerianaceae & $4,24^{* *}$ & 22,22 \\
\hline Calepina irregularis & Brassicaceae & $3,62^{* *}$ & 20,83 \\
\hline Geranium rotundifolium & Geraniaceae & $3,47^{* *}$ & 30,00 \\
\hline Barbarea intermedia & Brassicaceae & $2,82^{* *}$ & - \\
\hline Scandix pecten-venris & Apiaceae & $2,79 * *$ & 27,78 \\
\hline Carex hirta & Cyperaceae & $2,43^{*}$ & - \\
\hline Thlaspi arvense & Brassicaceae & $2,33^{* *}$ & 50,00 \\
\hline Anchusa arvensis & Boraginaceae & $2,10^{*}$ & - \\
\hline Geranium molle & Geraniaceae & $1,83^{* *}$ & 30,00 \\
\hline Reseda luteola & Resedaceae & $1,54^{* *}$ & - \\
\hline \multicolumn{4}{|l|}{ Repousses de cultures } \\
\hline Hordeum vulgare & Poaceae & $25,77^{* *}$ & - \\
\hline Triticum spp. & Poaceae & $25,23^{* *}$ & - \\
\hline Triticale & Poaceae & $1,98^{* *}$ & - \\
\hline
\end{tabular}

* Significatif à $p<0.05 .{ }^{* *}$ significatif à $p<0.01$.

\section{Évolution de la flore des communautés du colza : spécialisation ou banalisation?}

Nos résultats montrent que l'évolution des pratiques culturales tend, de manière générale, à favoriser une flore plus riche en espèces ubiquistes, tout en sélectionnant dans le même temps des espèces spécialistes du colza. Cette apparente contradiction se comprend si l'on admet que le processus d'évolution de la flore se joue à plusieurs échelles temporelles : (1) à court terme, au cours de la campagne où est cultivé le colza, mais aussi (2) à moyen terme, au cours de la rotation, et (3) à long terme, au fil de l'évolution globale des pratiques agricoles. Les processus actifs à chaque échelle (par exemple : les herbicides au sein du colza, le décalage ou non des cycles culturaux au cours de la rotation) ne sélectionnent pas forcément les mêmes caractères et donc les mêmes espèces (celles tolérantes aux herbicides du colza ou celles favorisées par des rotations longues). II demeure qu'un nombre plus élevé de parcelles présentent maintenant dans leur stock semencier des espèces propres à boucler leur cycle biologique quand ces parcelles sont cultivées en colza.

L'intensité des changements observée en 30 ans est incontestablement importante avec $28 \%$ de renouvellement spécifique au sein des 26 espèces majeures et l'extension rapide d'espèces comme Geranium dissectum, quasi inconnue des cultures de colza il y a 30 ans. Parmi les espèces en progression, plusieurs sont des hôtes de la plante parasite du colza Orobanche ramosa : Senecio vulgaris, Sonchus asper [14] ainsi que Lolium multiflorum, Geranium dissectum, Euphorbia helioscopia et Mercurialis annua $[15,16]$. En jouant le rôle de « réservoirs » secondaires de l'Orobranche rameuse, ces adventices ont permis à ce parasite de progresser en assurant son maintien dans les chaumes, jachères ou bord de champs.

\section{Influence des pratiques dans le colza}

Chaque fois que le colza est cultivé, les espèces spécialistes de cette culture (tableau 3) sont plus aptes que d'autres à produire des semences en raison entre autres du manque d'efficacité des programmes herbicides associés. Pour près de $70 \%$ de ces espèces, et en particulier pour les Brassicacées (Capsella bursa-pastoris, Calepina irregularis, Raphanus raphanistrum) et les Géraniacées (Geranium spp.), on peut en effet interpréter leur spécialisation marquée à la culture du colza comme le résultat d'un faible contrôle exercé par les herbicides [17]. Les dates de semis précoces, spécifiques à la culture de colza, sont aussi connues pour favoriser certaines espèces ayant un optimum de levée très précoce, dès la fin de l'été (Geranium spp., Scandix pecten-veneris, Valerianella spp.). Globalement, les conditions spécifiques au colza favorisant directement certaines espèces pourraient expliquer respectivement $30 \%$ à $50 \%$ des progressions nationale ou régionale. Parmi ces espèces spécialistes du colza, certaines, bien que n'apparaissant pas en progression au niveau national (Valerianella spp., C. irregularis, S. pecten-veneris ou encore Geranium molle) ont parfois progressé localement. Ces espèces ayant une amplitude agro-écologique plus stricte, leur progression reste toutefois limitée à certaines situations, - régions de plateaux argilo-calcaires pour Valerianella spp. et $S$. pecten-veneris ou plaines thermophiles pour $C$. irregularis - et n'est de ce fait pas décelable au niveau national.

\section{Influence des rotations dans lesquelles s'insère le colza}

Une situation de monoculture devrait théoriquement aboutir à la domination de quelques espèces «spécialistes ». Dans le cas du colza, la nature des autres cultures de la rotation devient déterminante pour contrôler ces espèces et influer sur la trajectoire de la communauté adventice hébergée. Dans l'Est, notamment dans les parcelles du groupe II, le colza s'insère principalement dans des rotations à base de cultures $d^{\prime}$ hiver « colza-blé-orge » (près de $75 \%$ des parcelles). Comme les autres cultures de la rotation ont un cycle hivernal comparable au colza, certaines espèces spécialistes du colza s'avèrent aussi adaptées à la phénologie des céréales d'hiver. Les espèces étant par ailleurs difficiles ( $B$. sterilis, Cirsium arvense) ou assez difficiles (Lolium spp., Geranium spp.) à désherber dans les céréales, seront alors d'autant plus rapidement favorisées dans ce type de succession culturale. Cela pourrait expliquer que le nombre d'espèces indicatrices du colza en progression ainsi que leur rang est supérieur dans ce type de rotation (groupe II) et plus généralement dans la région Est.

Dans l'Ouest, le colza prend place dans des rotations plus diversifiées incluant des cultures estivales et ne revient dans certains cas que tous les 4 ans (en particulier dans $71 \%$ des parcelles du groupe IV). Par rapport à l'Est, l'alternance de culture à cycle hivernal et estival pourrait par exemple être à l'origine d'un meilleur contrôle d'espèces hivernales comme $A$. myosuroides et de la progression moins nette des spécialistes du colza (G. dissectum, R. raphanistrum). En revanche, cette situation favoriserait des espèces plus ubiquistes, indifférentes à la nature de la culture (L. purpureum, Anagallis arvensis) ou plus ou moins spécialistes des cultures de printemps de la rotation (C. album, E. helioscopia, P. persicaria, $S$. vulgaris). Ces espèces progressent uniquement ou de façon plus nette dans l'Ouest. La présence d'espèces printanières dans le colza (C. album, M. annua, P. persicaria) serait aussi accentuée dans l'Ouest par un climat favorable. Si des espèces printanières très communes (et sans exigence climatique particulière) comme M. annua ou C. album peuvent se retrouver sur tout le territoire dans les cultures de printemps, il est a priori plus difficile pour ces espèces sensibles au gel de se développer dans une culture d'hiver comme le colza. La forte corrélation positive trouvée dans 
Tableau 4. Valeurs indicatrices des espèces pour les 4 principales situations agro-écologiques du colza en France. ** Significatif au risque $1 \%$, * au risque $5 \%$. Les valeurs indicatrices sont exprimées en \%.

\begin{tabular}{|c|c|c|c|c|c|}
\hline \multirow[t]{2}{*}{ Espèces } & \multicolumn{4}{|c|}{ Valeurs indicatrices (IndVal) } & \multirow[t]{2}{*}{ Fréquence } \\
\hline & I & II & III & IV & \\
\hline \multicolumn{6}{|l|}{ Inféodées au Groupe II } \\
\hline Alopecurus myosuroides & 10,23 & $23,85^{* *}$ & 6,37 & 3,29 & 27,0 \\
\hline Geranium dissectum & 7,98 & $30,58^{* *}$ & 5,06 & 10,57 & 17,2 \\
\hline Cirsium arvense & 2,41 & $18,53^{*}$ & 8,08 & 0,99 & 8,6 \\
\hline Geranium rotundifolium & 0,52 & $17,07^{* *}$ & 0,38 & 0,56 & 6,1 \\
\hline Bromus sterilis & 0,00 & $15,99 * *$ & 0,19 & 5,01 & 4,9 \\
\hline \multicolumn{6}{|l|}{ Inféodées au Groupe III } \\
\hline Daucus carota & 0,76 & 0,00 & $12,03^{* *}$ & 1,45 & 5,5 \\
\hline \multicolumn{6}{|l|}{ Inféodées au Groupe IV } \\
\hline Capsella bursa-pastoris & 5,69 & 13,54 & 4,70 & $24,00 * *$ & 27,6 \\
\hline Mercurialis annua & 3,13 & 0,43 & 6,20 & $39,52^{* *}$ & 26,4 \\
\hline Senecio vulgaris & 0,05 & 5,85 & 11,85 & $31,67^{* *}$ & 20,9 \\
\hline Chenopodium album & 3,11 & 0,26 & 9,50 & $28,84^{\star *}$ & 15,3 \\
\hline Poa annua & 1,41 & 0,59 & 5,93 & $21,71^{* *}$ & 12,3 \\
\hline Stellaria media & 5,13 & 1,93 & 2,42 & $17,90^{*}$ & 9,8 \\
\hline Lamium purpureum & 2,87 & 0,00 & 1,00 & $12,60^{*}$ & 8,6 \\
\hline Euphorbia helioscopia & 1,38 & 4,51 & 2,16 & $26,52^{* *}$ & 7,4 \\
\hline Picris hieracioides & 0,26 & 0,00 & 2,85 & $13,43^{* *}$ & 4,3 \\
\hline Calepina irregularis & 0,00 & 0,00 & 0,74 & $11,91^{\text {** }}$ & 3,7 \\
\hline \multicolumn{6}{|l|}{ Espèces indifférentes } \\
\hline Triticum spp. & 4,56 & 8,92 & 8,49 & 16,17 & 30,1 \\
\hline Viola arvensis & 1,32 & 12,69 & 12,55 & 16,31 & 28,2 \\
\hline Sinapis arvensis & 5,43 & 15,19 & 9,40 & 15,35 & 27,6 \\
\hline Lolium spp. & 5,75 & 9,11 & 18,00 & 9,04 & 19,6 \\
\hline Galium aparine & 4,51 & 7,26 & 17,52 & 6,93 & 19,0 \\
\hline Hordeum spp. & 8,85 & 11,42 & 7,22 & 2,30 & 19,0 \\
\hline Papaver rhoeas & 10,93 & 9,78 & 1,91 & 12,99 & 17,8 \\
\hline Sonchus asper & 6,91 & 11,68 & 7,95 & 7,42 & 16,0 \\
\hline Raphanus raphanistrum & 2,07 & 11,01 & 5,49 & 5,06 & 141 \\
\hline Myosotis arvensis & 2,16 & 6,68 & 7,79 & 4,16 & 13,5 \\
\hline Aphanes arvensis & 0,51 & 2,51 & 6,77 & 7,37 & 11,7 \\
\hline Veronica hederifolia & 0,21 & 10,02 & 4,32 & 15,15 & 11,0 \\
\hline Solanum nigrum & 2,78 & 1,57 & 6,43 & 4,74 & 9,8 \\
\hline Lapsana communis & 0,96 & 6,27 & 3,39 & 8,98 & 9,2 \\
\hline Polygonum persicaria & 0,35 & 3,38 & 2,79 & 7,95 & 8,6 \\
\hline Fumaria officinalis & 4,89 & 2,00 & 4,12 & 6,46 & 8,0 \\
\hline Sonchus oleraceus & 0,00 & 1,15 & 12,21 & 7,59 & 7,4 \\
\hline Ranunculus sardous & 0,47 & 0,00 & 2,76 & 9,45 & 6,7 \\
\hline Anagallis arvensis & 0,24 & 0,53 & 4,26 & 13,06 & 6,1 \\
\hline Polygonum aviculare & 4,36 & 0,00 & 6,40 & 2,93 & 6,1 \\
\hline Convolvulus arvensis & 0,33 & 7,94 & 3,90 & 2,86 & 5,5 \\
\hline Aethusa cynapium & 2,39 & 6,51 & 0,15 & 0,86 & 3,7 \\
\hline Chenopodium hybridum & 4,34 & 0,00 & 0,60 & 3,12 & 3,7 \\
\hline Chenopodium polyspermum & 1,55 & 1,27 & 0,00 & 1,68 & 3,7 \\
\hline Poa trivialis & 0,00 & 0,00 & 3,30 & 2,69 & 3,7 \\
\hline Taraxacum officinale & 0,65 & 2,82 & 2,38 & 0,93 & 3,7 \\
\hline Veronica arvensis & 0,60 & 5,24 & 0,59 & 2,89 & 3,7 \\
\hline
\end{tabular}

I'analyse de co-inertie entre C. album ou M. annua et la «température minimale en hiver » montrerait que c'est à la faveur des hivers doux que de telles espèces peuvent se développer et même parfois produire des graines avant l'hiver dans les colzas de ces régions. La présence de tournesol dans la rotation qui a en commun avec le colza $23 \%$ de matières actives herbicides pourrait aussi favoriser la progression dans le colza d'espèces qui sont à la fois ubiquistes et particulièrement adaptées au tournesol (E. helioscopia, S. vulgaris étaient par exemple toutes deux mal contrôlées par la trifluraline). La montée dans le colza d'espèces estivales (C. album, M. annua, P. persicaria, S. nigrum), soit au moins $30 \%$ 
des progressions, pourrait ainsi directement traduire l'influence de la rotation à travers l'augmentation des surfaces assolées en cultures estivales depuis 30 ans.

\section{Influence à long terme}

Depuis les années 1990, on observe un fléchissement des surfaces labourées lié à une diminution de la profondeur de travail du sol. L'analyse de co-inertie montre que les densités importantes de certaines espèces sont bien corrélées avec ce changement de pratique. Les espèces associées au non-labour semblent être essentiellement des espèces levant préférentiellement à partir des couches superficielles du sol $(0-5 \mathrm{~cm}): B$. sterilis, Viola arvensis, C. bursa-pastoris $[18,19]$. Des résultats semblables ont été observés pour la flore du colza en République Tchèque où $A$. spica-venti, B. sterilis, C. bursa-pastoris et Myosotis arvensis ont été trouvées comme associées aux techniques culturales simplifiés (T.C.S.) tandis que C. album y était comme dans le cas présent associée au labour [20]. En Allemagne, A. spica-venti, Viola arvensis, M. perforata semblent liées aux T.C.S. tandis que Lamium spp. décroît dans ces mêmes conditions [21] à l'instar de ce que l'on observe dans le colza en France. Dans une moindre mesure, des espèces disséminées par le vent comme Sonchus arvensis, Sonchus oleraceus, S. vulgaris ou Cirsium arvense, sembleraient également profiter du non-labour [22]. L'expansion du non-labour pourrait donc avoir contribué à faire progresser des espèces comme Viola arvensis, G. dissectum et G. rotundifolium ou encore S. vulgaris et Sonchus spp.

Outre l'effet de l'alternance des cultures d'hiver et de printemps, le phénomène de banalisation de la flore peut également s'expliquer à long terme par la régression des espèces spécialistes de milieux particuliers tels que sols siliceux-acides oligotrophes ou parcelles humides. Ainsi les régressions de Cerastium spp. et de Aphanes arvensis, plus ou moins accentuées selon les régions, peuvent résulter de l'uniformisation des conditions environnementales par chaulage des parcelles les plus acides ou amendements des parcelles les plus oligotrophes [23].

\section{Conclusion}

1) En 30 ans, $28 \%$ des 26 principales adventices de la flore du colza se sont trouvés renouvelés. Les espèces en progression sont soit des espèces plutôt ubiquistes (S. vulgaris, C. album) soit des espèces apparaissant comme inféodées au colza ( $G$. dissectum, $L$. communis).

2) Nous montrons que cette évolution résulte à la fois de processus opérant à l'échelle de la culture (tolérance aux herbicides du colza et concordance avec la date de semis), qu'aux échelles de la rotation (triant les spécialistes des cultures d'hiver, de printemps et les ubiquistes) et du long terme (réduction des surfaces labourées et banalisation des conditions environnementales).

3) À l'échelle de la France, le principal facteur actif sur la composition floristique des communautés résulterait des influences pédo-climatiques qui opposeraient la flore de I'Ouest à celle du Nord-Est. On observe logiquement des évolutions de flores différentes entre ces grandes régions. Dans l'Est, les conditions sont réunies pour une spécialisation plus rapide de la flore vis-à-vis du colza (rotations à base de culture d'hiver, retour plus fréquent du colza). Dans l'Ouest, l'effet des autres cultures de la rotation (maïs ou tournesol) et des rotations plus longues où le colza n'intervient qu'une fois sur 4 ans se combinent à un climat plus doux pour induire une progression plus rapide d'espèces estivales. 4) À l'échelle de la rotation, être une espèce spécialiste du colza peut certes être avantageux (possibilité de produire beaucoup de graines une année sur trois ou quatre), mais n'est ni une condition nécessaire ni une condition suffisante pour y réussir. À l'opposé, une espèce très ubiquiste est désarmée si par ailleurs elle est incapable de passer certains filtres du colza (date de semis ou herbicides) comme en témoignent les rangs dans le colza d'espèces généralistes comme Anagallis arvensis (32) ou Fallopia convolvulus (rang $>50)$. Si l'on s'en réfère à l'évolution globale de la communauté adventice (homogénéisation et spécialisation par rapport au colza), la réussite d'une espèce dans le colza devrait donc combiner des caractères lui permettant d'être suffisamment spécialiste du colza tout en restant assez ubiquiste pour passer dans les autres cultures et/ou se développer sur une vaste zone géographique. Ce cas de figure serait par exemple bien illustré par des espèces comme $C$. bursa-pastoris et Lolium spp. qui figurent aujourd'hui parmi les 10 principales adventices du colza. Ces espèces seraient en mesure de progresser encore à la différence de « vraies » spécialistes du colza qui pourraient être mieux contrôlées par l'introduction d'une culture estivale au cours de la rotation.

Remerciements. Les auteurs tiennent à remercier Marc Delos et toutes les personnes impliquées dans le fonctionnement du réseau Biovigilance Flore ainsi que Bruno Chauvel et Jacques Gasquez pour leurs relectures critiques du manuscrit. G. Fried bénéficie d'une formation complémentaire par la recherche (FCPR) du ministère de l'Agriculture et de la Pêche.

\section{RÉFÉRENCES}

1. KROPAC Z, HADAC E, HEJNY S. Some remarks on the synecological and Syntaxonomic Problems of Weed Plant Communities. Preslia 1971; 43 : 139-53.

2. BOOTH BD, SWANTON C). Assembly theory applied to weed communities. Weed Sci $2002 ; 50: 2-13$

3. FRIED G, CHAUVEL B, REBOUD X, CHOLLET D, BOMBARDE M, DELOS M. Évolution de la flore adventice en 30 ans : quelles caractéristiques semblent favoriser la capacité d'infestation en tournesol? Phytoma-LDV 2006 ; 596 : 37-43.

4. BARRALIS G. Répartition et densité des principales mauvaises herbes en France. Document INRA-AFPP. 1977 ; (1-22).

5. DELOS M, HERVIEU F, FOLCHER L, MICOUD A, LA EYCHENNE N. «Biovigilance ", des OGM au général. Exemple du suivi des grandes cultures en France. Phytoma-LDV 2006 ; 589 : 44-8.

6. BARRALIS G. Méthode d'étude des groupements adventices des cultures annuelles. In : Vè Colloque International sur l'Ecologie et la Biologie des Mauvaises herbes, 1, Dijon, France. $1976:$ 59-68.

7. BENICHOU P, LEBRETON O. Prise en compte de la topographie pour la cartographie des champs pluviométriques statistiques. La météorologie $1987 ; 7: 23-34$; (et 14 cartes encartées hors texte).

8. BRISSE H, DE RUFFRAY P, GRANDJOUAN G, HOFF M. La banque de données phytosociologiques "SOPHY". In : Proceedings of the 4th International workshop" European vegetation survey". Ann di Bot, Vol. LIII, 1995, Roma, Italy. $1996: 177-223$

9. DUFRÊNE M, LEGENDRE P. Species assemblages and indicator species : the need for a flexible asymmetrical approach. Ecol Monogr 1997 ; 67 : 345-66.

10. HILL MO, SMITH AJE. Principal component analysis of taxonomic data with multi-state discrete characters. Taxon $1976 ; 25: 249-55$.

11. WARD JH. Hierarchical grouping to optimize an objective function. / Am Stat Assoc $1963 ; 58: 238-44$.

12. BORNETTE G, AMOROS A, CHESSEL D. Rejuvenation in former braided channels of the Rhône River : successional patterns and allogenic processes. I Veg Sci $1994 ; 5$ : 237-46.

13. THIOULOUSE J, CHESSEL D, DOLÉDEC S, OLIVIER JM. ADE-4 : a multivariate analysis and graphical display software. Stat Comput $1997 ; 7(1)$ : 75-83.

14. BOULET C, LABROUSSE P, ARNAUD MC, ZEHHAR N, FER A. Weed species present various responses to Orobanche ramosa. In : Fer A, Thalouarn $\mathrm{P}$, Joel DM, Musselman LJ, Parker C, Verkleij JAC, eds. Proceedings of the seventh international parasitic Weed Symposium. Faculté des Sciences de Nantes. 2001 : 228-31. 
15. GIBOT-LECLERC S, BRAULT M, PINOCHET X, SALLE G. Rôle potentiel des plantes adventices du colza d'hiver dans l'extension de l'orobanche rameuse en Poitou-Charentes. Comptes Rendus Biologies Paris 2003 ; 326 : 645-58.

16. GIBOT-LECLERC S, PINOCHET X, SALLE G. Orobanche rameuse (Orobanche ramosa L. ) du colza : un risque émergent sous surveillance. OCL 2006 ; 13 : 200-5.

17. MAMAROT J, RODRIGUEZ A. Sensibilité des mauvaises herbes aux herbicides en grandes cultures (1st ed.). Paris : ACTA, 2003.

18. JAUZEIN P, VACHER C, BLONDLOT A, CITRON G. Les bromes en extension dans les céréales. Perspectives Agricoles $2000 ; 259: 82-92$.

19. ZAWIEJA J, KORDAS L. Effect of simplified tillage and direct sowing on weed seedbank in soil. Acta Scientiarum Polonorum - Agricultura 2003; 2(2) : 163-70.
20. WINKLER J, ZELENA V. Impact of different soil cultivation on weed species in winter rape (oilseed). Acta Universitatis Agriculturae et Silviculturae Mendelianae Brunensis 2005 ; 53(5) : 187-94.

21. PALLUTT B. Influence of crop rotation, tillage and herbicide use on population dynamics and competition of weeds in winter cereals. Gesunde Pflanzen $1999 ; 51(4)$ : 109-20.

22. DERKSEN DA, LAFOND GP, THOMAS AG, LOEPPKY HA, SWANTON C). Impact of Agronomic Practices on Weed Communities: Tillage Systems. Weed Sci $1993 ; 41: 409-17$.

23. JAUZEIN P. L'appauvrissement floristique des champs cultivés. Dossier de I'environnement de I'INRA $2001 ; 21: 65-78$. 